\title{
Evaluation of a modified Rose Bengal test and an indirect Enzyme-Linked Immunosorbent Assay for the diagnosis of Brucella melitensis infection in sheep
}

\author{
Ana Cristina FERrEIRA ${ }^{\mathrm{a}}$, Regina CARDOSO ${ }^{\mathrm{a}}$, Isabel TrAVASSOS DiAS ${ }^{\mathrm{a}}$, \\ Isabel MARIANO ${ }^{b}$, Ana BELO ${ }^{c}$, Isabel RoLÃO PRETO ${ }^{c}$, António \\ MANTEIGAS $^{\mathrm{c}}$, António PINA FonsECA ${ }^{\mathrm{c}}$, Maria Inácia CORRÊA DE SÁa ${ }^{\text {* }}$ \\ a Laboratório Nacional de Investigação Veterinária (LNIV), Estrada de Benfica, \\ 701,1549-011 Lisboa, Portugal \\ b Laboratório Veterinário de Montemor-o-Novo- COPRAPEC, 7050 Montemor-O-Novo, Portugal \\ c Direcção Regional de Agricultura da Beira Interior (DRABI), 6001 Castelo Branco, Portugal
}

(Received 9 August 2002; accepted 9 December 2002)

\begin{abstract}
A modified Rose Bengal test (mRB) and an indirect ELISA (iELISA) with Protein G as the conjugate, were evaluated for the diagnosis of Brucella melitensis infection in unvaccinated sheep with a known bacteriological status, and their diagnostic efficacy was compared with that of the standard Rose Bengal (RB) and Complement Fixation (CF) tests used in the current eradication campaign in EU countries. All tests showed $100 \%$ specificity when testing the sera from 212 Brucella-free sheep. When testing the sera from 219 Brucella melitensis culture-positive sheep, both the $\mathrm{mRB}$ and iELISA tests were more sensitive $(98.6 \%$ and $96.8 \%$, respectively) than the RB and CF tests (95.0\% and $92.7 \%$, respectively). These results were similar when testing the sera from 181 animals belonging to infected flocks but found bacteriologically negative, suggesting that the mRB or iELISA tests could advantageously replace the current RB procedure used as the screening test.
\end{abstract}

sheep / Brucella melitensis / serological diagnosis / modified rose bengal test / indirect ELISA

\section{INTRODUCTION}

Brucellosis is an important infectious disease mainly affecting cattle, sheep and goats. The disease in cattle is most commonly caused by Brucella abortus whilst brucellosis in sheep and goats, a disease highly prevalent in southern EU countries, is caused by Brucella melitensis, a very important zoonotic agent [12, 15, 25, 29].

The diagnostic method known to produce the best results in terms of specificity is the isolation of Brucella organisms from the suspected animal. However, this method has a limited sensitivity, is expensive and cumbersome and has the added difficulty of being unpractical to apply at a large scale in control campaigns. Accordingly, the indirect diagnosis of disease based on serological tests is of choice in the eradication programmes. The standard Rose Bengal (RB) and Complement Fixation (CFT) tests are the main serological tests used to detect antibodies against

\footnotetext{
* Correspondence and reprints

Tel.: (351) 217115223; fax: (351) 217115383; e-mail: inacia.sa@lniv.min-agricultura.pt
} 
B. abortus and B. melitensis infections. Both tests have been used for several decades, proving to be successful for eradicating bovine brucellosis in some countries, and are the official tests currently used in the EU for the eradication of B. melitensis infection in small ruminants. Nevertheless, there is evidence that both tests are significantly less effective for the diagnosis of brucellosis in sheep and goats than in cattle $[12,15]$.

The EU rules consider that the best strategy for diagnosing sheep and goat brucellosis is the combined use of RB as the screening test and the $\mathrm{CF}$ as the confirmatory test. However, it has been reported that a relatively high proportion of sheep and goats from $B$. melitensis-infected flocks show negative results in the standard $\mathrm{RB}$ test but positive in the $\mathrm{CF}$ test, questioning the sensitivity of the former as a screening test [4]. Thus, the simultaneous use of both is recommended to obtain a maximal sensitivity $[4,12]$. To improve the sensitivity of the RB test a simple modification consisting in increasing the volume of sera to be tested has been recommended [4, 5]. This RB modified test (mRB) significantly increases the sensitivity of the RB standard procedure and considerably reduces the problem of sera being $\mathrm{RB}$ negative but $\mathrm{CF}$ positive (Blasco J.M., personal communication). During recent years, different indirect EnzymeLinked Immunosorbent Assays (iELISAs) have been developed using more or less purified S-LPS as the antigen and have been reported to be at least as sensitive and specific as the combination of both RB and $\mathrm{CF}$ tests for the diagnosis of brucellosis in ruminants $[1,6,11,17-19,22-24,28,30]$. In fact, an iELISA with polyclonal conjugate (anti-IgG $\mathrm{H}+\mathrm{L}$ ) has been reported to be effective enough for the diagnosis of the infection in sheep and goats [5, 7, 14]. However, one of the problems of this test is that the conjugate used results in a high background reactivity when testing sera from Brucella-free animals, reducing the specificity of the test [14]. The use of Pro- tein $\mathrm{G}$ as the conjugate reduces this problem increasing ELISA specificity [9, 22], with the additional advantage of protein $G$ being a suitable reagent in enzyme immunoassays designed for all domestic ruminant species [22].

In Portugal, a complex strategy with the sequential or parallel use of the RB and CFT was established, depending on the sanitary classification of the flock (Norms for the Laboratory Analysis of Eradication Plans for Brucellosis, Agriculture Ministry, DGV, Circular No. 80/DSSA, 14/06/00). In the case of Brucella-Free flocks, confirmation of each positive sera in the RB test has to be done by the CF test. In Brucella infected flocks, however, the RB test is used directly as a confirmatory test. If herds being tested for a long time (more than 24 months) remain having RB positive animals, all sera being RB negative are also tested by the CF test. These procedures and test combinations complicate a lot the official eradication campaign and considerably increase their costs, justifying the search of tests of increased sensitivity and/or specificity. The aims of the present work were: (i) to compare the diagnostic performance of both $\mathrm{mRB}$ and iELISA / protein G in sheep with a known bacteriological status with that of the standard RB and CF official tests in the $\mathrm{EU}$, and (ii) to determine the diagnostic performance of both tests in infected flocks having animals positive in the classical RB and/or CF test, but in which $B$. melitensis could not be isolated.

\section{MATERIALS AND METHODS}

\subsection{Samples}

The sheep sera used in this study were collected at slaughter from 400 unvaccinated animals belonging to several flocks in which $B$. melitensis infection was bacteriologically proven. Fragments from the liver, spleen, uterus (when the animals 
were pregnant) and the whole submaxillary and supramammary lymph nodes were collected to assess the bacteriological status of the animals.

Samples of the intestine (ileon) were also collected from all the animals for the assessment of the eventual existence of the cross-reacting bacteria such as Escherichia coli O:157, Salmonella spp. and Yersinia enterocolitica 0:9. The procedures and culture media used for this purpose have been described elsewhere [3, 8, 27].

Additionally, sera from 212 sheep belonging to Brucella-free flocks were collected as negative controls.

\subsection{Bacteriological procedures}

Culture and identification of Brucella spp. was carried out by standard procedures. Briefly, each sample was homogenised in sterile tryptose saline solution and $0.2 \mathrm{~mL}$ of each homogenate was smeared on each of 6 plates of the modified Farrell medium [10]. This medium was prepared with tryptose agar (Difco, Detroit, Michigan, USA) with 5\% sterile equine serum and a commercial freeze-dried selective supplement (Oxoid, DuPont, Qualicon Inc., USA). In $1 \mathrm{~L}$ of the medium there was nalidixic acid $5 \mathrm{mg}$, bacitracin $25000 \mathrm{IU}$, natamycin $50 \mathrm{mg}$, polymixin B sulphate $5000 \mathrm{IU}$, vancomycin $20 \mathrm{mg}$ and nystatin 100000 IU. The plates were incubated for up to 10 days, at $37^{\circ} \mathrm{C}$, half of them in a normal atmosphere and the other half in a 5\% $\mathrm{CO}_{2}$ atmosphere. The presumptive identification of Brucella melitensis was conducted on the basis of colony morphology, Gram staining and biochemical tests $\left(\mathrm{CO}_{2}\right.$ requirement, catalase, oxidase, urease, dye sensitivity and $\mathrm{H}_{2} \mathrm{~S}$ production). Biovars were identified by agglutination with monospecific anti-A and anti-M sera [2].

According to the bacteriological results, the following groups of sera were obtained:

(i) The infected group composed of sera from 219 sheep in which B. melitensis was isolated. (ii) The suspected group composed of sera from 181 sheep that were culturenegative but belonged to flocks in which $B$. melitensis was confirmed bacteriologically.

(iii) The Brucella-free group composed of sera from 212 sheep belonging to Brucella-free flocks.

\subsection{Serological tests}

\subsubsection{Antigen Preparations}

The antigens used for the Rose Bengal and Complement Fixation tests were prepared according to Hendry et al. [13], from Brucella abortus biovar 1, strain 99 (Weybridge, United Kingdom) and standardised according to EU requirements. For ELISA, the crude smooth Lipopolysaccharide (S-LPS) extract was used, prepared from Brucella abortus biovar 1, strain 99 (Weybridge, United Kingdom) by a phenolwater extraction according to Hendry et al. [13].

\subsubsection{Rose Bengal (RB) test}

The RB test was performed, following the procedure described by Alton et al. [2]. The plates were shaken for 4 min and any agglutination that appeared within this time was recorded as a positive reaction.

\subsubsection{Modified Rose Bengal (mRB) test}

This test was performed following the procedure described by Blasco et al. [4], mixing $75 \mu \mathrm{L}$ of sera and $25 \mu \mathrm{L}$ of the antigen. The plates were shaken for $4 \mathrm{~min}$ and any agglutination that appeared within this time was recorded as a positive reaction.

\subsubsection{Complement Fixation (CF) test}

The CFT was performed on a microplate, following the "warm" procedure described by Alton et al. [2]. 
The sera were considered positive if they showed at least $50 \%$ hemolysis at a $1 / 4$ dilution (i.e. $\geq 20$ ICFTU).

\subsubsection{Indirect ELISA (iELISA)}

The iELISA was performed as described previously $[12,14]$ with some modifications. Briefly, standard 96-well polystyrene plates (Dynatech M129B) were coated at $4{ }^{\circ} \mathrm{C}$ overnight with S-LPS $(0.4 \mu \mathrm{g} / \mathrm{mL} ; 100 \mu \mathrm{L}$ per well $)$ suspended in $10 \mathrm{mM}$ Phosphate Buffered Saline (PBS) (pH 7.2). Non-absorbed antigen was removed by four washings with PBS containing $0.05 \%$ Tween-20 (PBST). The sera were diluted to $1 / 200$ in PBST. $100 \mu \mathrm{L}$ of each dilution were added in duplicate and the plates were incubated for $1 \mathrm{~h}$ at $37^{\circ} \mathrm{C}$ and were washed four times with PBST. The protein $\mathrm{G}$ conjugate $(0.125 \mu \mathrm{g} / \mathrm{mL}$, P-8170; Sigma, St. Louis, MO, USA) was added $(100 \mu \mathrm{L}$ per well) and the plates were incubated for $1 \mathrm{~h}$ at $37^{\circ} \mathrm{C}$ and washed four times with PBST. Finally, $100 \mu \mathrm{L}$ of the substrate solution (10 mg $o$-phenylenediamine (OPD, P-8287, Sigma, St. Louis, MO, USA)) in $25 \mathrm{~mL}$ of $0.05 \mathrm{M}$ Phosphate-Citrate Buffer $\mathrm{pH}$ 5.0, with $10 \mu \mathrm{L}$ of $\mathrm{H}_{2} \mathrm{O}_{2}$ ) were added and the optical density (OD) was determined at $492 \mathrm{~nm}$ in a Dynex MRX Microplate reader (Dinex Technologies Inc., Chantilly, VA, USA) after $15 \mathrm{~min}$ of incubation at room temperature in the absence of light. Two positive and two negative sera control tests were repeated in all plates.

The optimal antigen concentration and conjugate dilution were determined in a preliminary experiment by testing 20 sera from culture-positive animals and 20 sera from Brucella-free animals. The optimal serum dilution was determined by testing positive sera from Brucella culture-positive animals showing 213 ICFTU (International Complement Fixation Test Units) and negative sera from Brucella-free sheep, both diluted in PBST from 1/100 to $1 / 102400$.

\subsection{Statistics}

Data were compiled in a database and separated into negative or positive according to the results of the different serological tests.

For iELISA, the optimal cut-off value was determined by receiver operating characteristics (ROC) analysis (Win Episcope, version 2.0).

Test agreement (Kappa values) was calculated using standard procedures (Win Episcope, version 2.0).

\section{RESULTS}

\subsection{Bacteriological results}

A total of 219 animals out of the 400 examined were culture positive. The isolated strains corresponded to Brucella melitensis biovar 3 in all cases. There was no isolation of cross-reacting bacteria in any of the corresponding 400 intestinal samples examined bacteriologically.

\subsection{Serological results}

The distribution of the OD values of the Brucella melitensis culture-positive and Brucella free populations in the iELISA is represented in Figure 1. As it can be seen, the $B$. melitensis infected animals were not fully discriminated from the Brucella-free ones. However, the mean OD value \pm SD for the sera from the Brucella-free animals was $0.060 \pm 0.046$ and this value was $1.675 \pm 0.562$ for the sera from Brucella melitensis culture-positive animals.

The optimal cut-off value after ROC analysis (resulting in $96.8 \%$ sensitivity and $100 \%$ specificity) was 0.250 . The same OD distribution for the animals from the suspected group is also represented in Figure 1 and as it can be seen, this OD distribution was practically identical to that described for the culture positive animals. 


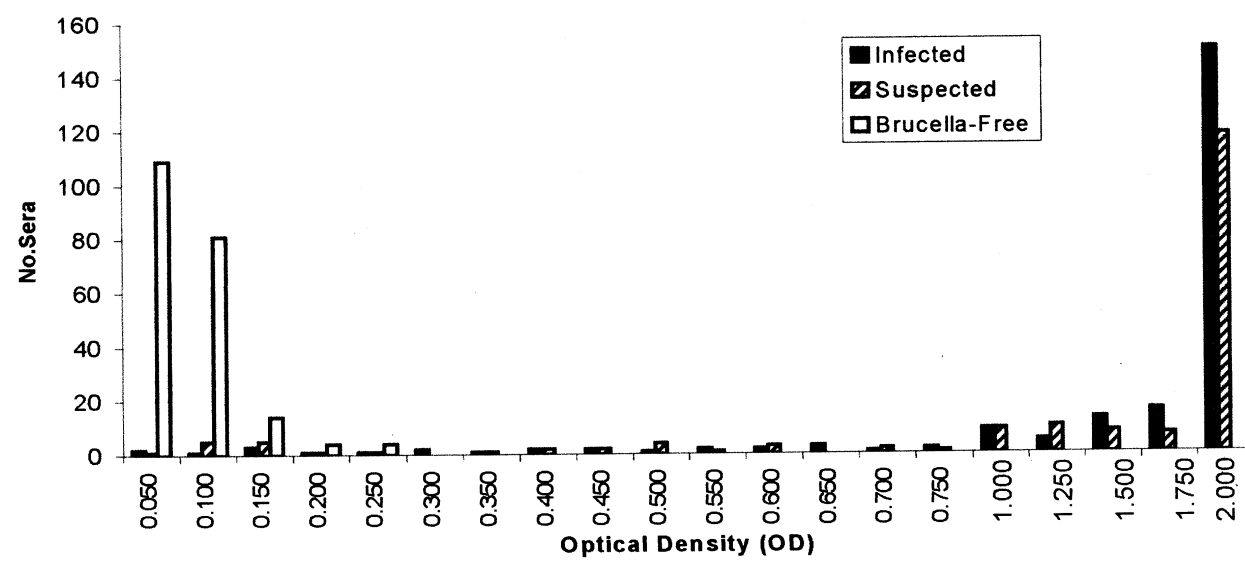

Figure 1. Distribution of iELISA OD values when testing the 219 sera from B. melitensis-infected animals, the 181 sera from suspected sheep and 212 sera from Brucella-free animals.

For comparative purposes, the sensitivity was calculated as the percentage of culture positive animals identified as positive in a given test and the specificity was defined as the percentage of Brucella-free animals identified as negative in the corresponding test. The best sensitivity results (Tab. I; group I) were obtained with the mRB (98.6\%) and iELISA (96.8\%). Both the $\mathrm{CF}$ and the standard $\mathrm{RB}$ were significantly $(P<0.005)$ less sensitive $(92.7 \%$ and $95.0 \%$, respectively) than both $\mathrm{mRB}$ and iELISA. All tests resulted in 100\% specificity when testing the sera from the animals belonging to the Brucella-free flocks (Tab. I; group III animals).

The results in terms of apparent sensitivity (i.e., the percentage of suspected animals with positive results in a given test) obtained in the different tests with sera from the 181 culture negative animals belonging to infected flocks (suspected group II) are also presented in Table I. The apparent sensitivity of both $\mathrm{mRB}$ and iELISA were identical (92.8\%) and in both cases significantly higher $(P<0.005)$ than that of both the standard RB and CF tests (87.8\% and $87.3 \%$, respectively).

Table I. Results of the serological tests when testing sera from B. melitensis infected (Group I), suspected (Group II), and Brucella-free (Group III) sheep.

\begin{tabular}{|c|c|c|c|c|c|c|c|c|c|}
\hline \multirow[b]{2}{*}{ Groups } & \multirow{2}{*}{$\begin{array}{c}\text { No. } \\
\text { animals }\end{array}$} & \multicolumn{2}{|c|}{$\mathrm{RBm}$} & \multicolumn{2}{|c|}{ iELISA $^{a}$} & \multicolumn{2}{|c|}{$\mathrm{RB}^{\mathrm{a}}$} & \multicolumn{2}{|c|}{$\mathrm{CFT}^{\mathrm{a}}$} \\
\hline & & Pos. & Neg. & Pos. & Neg. & Pos. & Neg. & Pos. & Neg. \\
\hline I & & & & & & & & & \\
\hline Infected & 219 & $\begin{array}{c}216^{\mathrm{a}} \\
(\mathbf{9 8 . 6})\end{array}$ & $\begin{array}{c}3 \\
(1.4)\end{array}$ & $\begin{array}{c}212 \\
(\mathbf{9 6 . 8})\end{array}$ & $\begin{array}{c}7 \\
(3.2)\end{array}$ & $\begin{array}{c}208 \\
(\mathbf{9 5 . 0})\end{array}$ & $\begin{array}{c}11 \\
(\mathbf{5 . 0})\end{array}$ & $\begin{array}{c}203 \\
(\mathbf{9 2 . 7})\end{array}$ & $\begin{array}{c}16 \\
(7.3)\end{array}$ \\
\hline II & & & & & & & & & \\
\hline Suspected & 181 & $\begin{array}{c}168 \\
(\mathbf{9 2 . 8})\end{array}$ & $\begin{array}{c}13 \\
(7.2)\end{array}$ & $\begin{array}{c}168 \\
(\mathbf{9 2 . 8})\end{array}$ & $\begin{array}{c}13 \\
(7.2)\end{array}$ & $\begin{array}{c}159 \\
(\mathbf{8 7 . 8})\end{array}$ & $\begin{array}{c}22 \\
(\mathbf{1 2 . 2})\end{array}$ & $\begin{array}{c}158 \\
(\mathbf{8 7 . 3})\end{array}$ & $\begin{array}{c}23 \\
(\mathbf{1 2 . 7})\end{array}$ \\
\hline $\begin{array}{l}\text { III } \\
\text { Brucella-free }\end{array}$ & 212 & 0 & $\begin{array}{c}212 \\
(\mathbf{1 0 0})\end{array}$ & 0 & $\begin{array}{c}212 \\
(\mathbf{1 0 0})\end{array}$ & 0 & $\begin{array}{c}212 \\
(\mathbf{1 0 0})\end{array}$ & 0 & $\begin{array}{c}212 \\
(\mathbf{1 0 0})\end{array}$ \\
\hline
\end{tabular}

a Number (\%). (RB: standard Rose Bengal test; mRB: modified Rose Bengal test; iELISA: indirect ELISA with S-LPS as the antigen and protein G as the conjugate; CFT: Complement Fixation test). 
Table II. Agreement (Kappa values) between the several serological tests when testing sera from culture positive $(n=219)$ and Brucella-free animals $(n=212)$.

\begin{tabular}{|c|c|c|c|c|c|c|c|c|c|}
\hline & CFT + & CFT - & Kappa & $\mathrm{RBm}+$ & $\mathrm{RBm}-$ & Kappa & iELISA + & iELISA - & Kappa \\
\hline $\mathrm{RB}+$ & 197 & 11 & \multirow{2}{*}{0.921} & 208 & 0 & \multirow{2}{*}{0.963} & 205 & 3 & \multirow{2}{*}{0.954} \\
\hline $\mathrm{RB}-$ & 6 & 217 & & 8 & 215 & & 7 & 216 & \\
\hline $\mathrm{RBm}+$ & 202 & 14 & \multirow{2}{*}{0.930} & & & & & & \\
\hline $\mathrm{RBm}-$ & 1 & 214 & & & & & & & \\
\hline iELISA + & 201 & 11 & \multirow{2}{*}{0.940} & 211 & 1 & \multirow{2}{*}{0.972} & & & \\
\hline iELISA - & 2 & 217 & & 5 & 214 & & & & \\
\hline
\end{tabular}

Table III. Agreement (Kappa values) between the several serological tests when testing sera from the suspected animals $(n=181)$ and Brucella-free animals $(n=212)$.

\begin{tabular}{lccccccccc}
\hline & CFT + & CFT - & Kappa & RBm + & RBm - & Kappa & iELISA + & iELISA - & Kappa \\
\hline $\mathrm{RB}+$ & 147 & 12 & \multirow{2}{*}{0.878} & 159 & 0 & 0.953 & 154 & 5 & 0.901 \\
$\mathrm{RB}-$ & 11 & 223 & & 9 & 225 & & 14 & 220 & \\
$\mathrm{RBm}+$ & 152 & 16 & & & & & & & \\
$\mathrm{RBm}-$ & 6 & 219 & 0.885 & & & & & & \\
$\mathrm{iELISA}+$ & 155 & 13 & & 163 & 5 & & & & \\
iELISA - & 3 & 222 & 0.916 & 5 & 220 & 0.948 & & & \\
\hline
\end{tabular}

The Kappa values of the different tests were calculated when testing the sera from culture positive and Brucella-free animals (Tab. II) and from suspected and Brucellafree animals (Tab. III). The agreement between the tests was generally high. When comparing culture positive and Brucella-free animals, iELISA and $\mathrm{mRB}$ showed the highest agreement (0.972; Tab. II). The highest agreement (Kappa = 0.953 ) when comparing the suspected and Brucella free animals was found among $\mathrm{RB}$ and $\mathrm{mRB}$ tests (Tab. III).

\section{DISCUSSION}

As in other EU countries [4], in the current $B$. melitensis eradication programme conducted in Portugal, a relatively high percentage of sheep which are negative in the RB screening test but positive in the $\mathrm{CF}$ confirmatory test has been identified.
As seen in Tables II and III, figures as high as 3\% (in infected animals) and 7\% (in suspected animals) resulted in RB negative but CF positive. In fact, several EU countries including Portugal are simultaneously applying both tests to increase sensitivity in the current $B$. melitensis eradication programme. This strategy can lead to a considerable lack of specificity in countries using Rev1 vaccination in replacement animals or affected by the false positive serological reactions due to cross-reacting bacteria, a problem that also seems to affect sheep and goats $[12,16]$. Moreover, this test combination is very expensive, time consuming and represents a complication of importance for most EU countries applying the official B. melitensis eradication programme in small ruminants.

The RB plate agglutination test antigen used in the EU is standardised without reference to the cell concentration, in such a way that a positive reaction can be 
achieved at a dilution of $1 / 47.5$ with the OIEISS reference serum and a negative reaction can be obtained at a dilution of $1 /$ 55 of this same serum [26].

In fact, it has been clearly shown that this antigen standardisation procedure significantly limits the sensitivity of the RB test in sheep and goats and explains the existence of many animals that are negative in the RB test but positive in the CF test, particularly in moderate to low prevalence conditions [4]. Suitable alternatives could be based on increasing the sensitivity limits against the OIEISS without affecting the specificity or, alternatively, modifying the antigen standardisation procedure using a wide sera panel of $B$. melitensis culturepositive and Brucella-free animals rather than a single reference serum [4]. Moreover, the current standard RB test procedure using equal volumes of serum and antigen is considered to be suitable (despite no rigorous existing evidence) for the diagnosis of $B$. abortus infection in cattle, but is not sensitive enough for $B$. melitensis diagnosis in sheep [4]. In fact, these authors described a modified RB test procedure $(\mathrm{mRB})$, using three volumes of sera $(75 \mu \mathrm{L})$ and one $(25 \mu \mathrm{L})$ of the RB antigen, that significantly increased the sensitivity of the European RB antigens without affecting their specificity. In agreement with this finding, in the particular epidemiological conditions of Portugal, this simple RB test modification significantly increased the sensitivity of the RB test without affecting the specificity (Tab. I). Moreover, this modification significantly reduced the number of negative sera in the RB test but positive in the CF test (Tabs. II and III). All tests evaluated showed $100 \%$ specificity when testing sera from Brucella free animals and, in general, a high sensitivity for detecting culture-positive animals. The $\mathrm{mRB}$ and iELISA were the most sensitive tests and gave the higher proportion of agreement (Tab. II). The CF was the less sensitive test $(92.7 \%)$ followed by the standard RB $(95 \%)$. These results are in agreement with those previously reported by others $[4,12]$; and seriously question the value of the current diagnostic strategy applied for eradicating $B$. melitensis in sheep and goats in the EU countries. Considering the higher sensitivity of the $\mathrm{mRB}$ with respect to the $\mathrm{RB}$ and that the problem of $\mathrm{RB}$ negative but $\mathrm{CF}$ positive sera was almost totally abrogated by the use of mRB (only one of 219 sera from culture positive sheep was $\mathrm{mRB}$ negative but CF positive, Tab. II), this simple $\mathrm{RB}$ test modification could help to reduce costs and complications in the current eradication campaigns conducted in the EU countries. However, like the standard RB, the $\mathrm{mRB}$ test has some degree of subjectivity, is slow and cumbersome to perform and not easily automated. Thus, the search for alternative screening tests is recommended with the iELISA being one of the best candidates. The sensitivity obtained $(96.8 \%)$ with our iELISA was somewhat lower than that reported by others $[1,23]$. Moreover, and in contrast with other experiments resulting in a better discrimination between the culture positive and Brucella-free populations $[9,12,21]$, our sera from culturepositive animals were not clearly discriminated from those of Brucella-free sheep (Fig. 1). Small differences in the test reagents and standardisation conditions could account for these small discrepancies. The similar sensitivity results obtained with the $\mathrm{mRB}$ and iELISA suggest that either of these tests could be used in replacement of the standard RB test.

For culture negative animals belonging to infected flocks (suspected group II), the results were somewhat different to those obtained with culture positive animals (Tabs. I, II and III). Again, the $\mathrm{mRB}$ and iELISA showed the same sensitivity and were more sensitive than both standard tests in these suspected sera. The existence of serologically positive but culture negative sheep is a relatively frequent finding in infected flocks. In the case of Rev 1 vaccinated animals, this positive serological response in the absence of infection could be due to a secondary immunological response as a consequence of antigenic 
contacts with field B. melitensis strains. However, vaccination was not performed in any of the flocks selected in our study. The existence of false positive serological reactions, a phenomenon of increasing importance in small ruminants [12], could also be another possibility. However, no relevant cross-reacting bacteria were isolated, even in the cultured intestinal samples of the 400 slaughtered sheep. Accordingly, a low level of sensitivity of the bacteriological procedures used could be the most probable reason to explain the existence of these 181 serologically positive but culture negative animals belonging to infected flocks. For a proper sensitivity for the isolation of $B$. melitensis from sheep, many organs and lymph nodes have to be sampled [20] and in our study only a few were sampled. Accordingly, most of these 181 suspected animals could have had true infections that were not properly detected. In fact, the serological results in these suspected animals were highly correlated with those obtained in culture positive sheep (Tabs. II and III; Fig. 1).

In conclusion, the mRB test could be a simple and economic alternative to advantageously replace the standard RB test procedure for the diagnosis of $B$. melitensis infection in the current epidemiological conditions of Portugal and many other EU countries.

In countries in which no vaccination programmes are being used, the $\mathrm{CF}$ test could be replaced by either the $\mathrm{mRB}$ or iELISA to increase diagnostic sensitivity.

\section{ACKNOWLEDGEMENTS}

The authors wish to thank Teresa Albuquerque and Patricia Temudo, from the Bacteriological Department of LNIV, Portugal.

This work was supported by a grant from the Instituto Nacional de Investigação Agrária (INIA) -PAMAF 3002. Ana Cristina Ferreira and Regina Cardoso were supported by a research-training grant from this same Institute.

\section{REFERENCES}

[1] Alonso-Urmeneta B., Marín C., Aragón V., Blasco J.M., Díaz R., Moriyón I., Evaluation of Lipopolysaccharides and Polysaccharides of different Epitope Structures in the Indirect Enzyme-Linked Immunosorbent Assay for Diagnosis of Brucellosis in Small Ruminants and Cattle, Clin. Diagn. Lab. Immunol. 5 (1998) 749-754.

[2] Alton G.C., Jones L.M., Angus R.D., Verger J.M., Techniques for the Brucellosis Laboratory, Institut National de la Recherche Agronomique, Paris, France, 1988.

[3] Bergey's Manual of Systematic Bacteriology. Enterobacteriaceae - Yersinia, Vol. 1. Ed. Williams \& Wilkins, Baltimore, London, 1984, pp. 498-506.

[4] Blasco J.M., Garin-Bastuji B., Marín C., Gerbier G., Fanlo J., Jiménez De Bagués M., Cau C., Efficacy of different Rose Bengal and Complement Fixation antigens for the diagnosis of Brucella melitensis in sheep and goats, Vet. Rec. 134 (1994) 415-420.

[5] Blasco J.M., Marín C., Jiménez De Bagués M., Barberán M., Hernández A., Molina L. Velasco J., Díaz R., Moriyón I., Evaluation of allergic and serological tests for diagnosing Brucella melitensis infection in sheep, J. Clin. Microbiol. 32 (1994) 1835-1840.

[6] Cloeckaert A., Weynants V., Godfroid J. Verger J.M., Grayon M., Zygmunt M.S., O-Polysaccharide epitopic heterogeneity at the surface of Brucella spp. studied by Enzyme-Linked Immunosorbent Assay and Flow Cytometry, Clin. Diagn. Lab. Immunol. 5 (1998) 862-870.

[7] Díaz-Aparício E., Marín C., AlonsoUrmeneta B., Aragón V., Perez-Ortiz S., Pardo M., Blasco J.M., Díaz R., Moriyón I., Evaluation of serological tests for diagnosis of Brucella melitensis infection of goats, J. Clin. Microbiol. 32 (1994) 1159-1165.

[8] Edwards and Ewing's, The Genus Yersinia, in: Identification of Enterobacteriaceae, 4th ed., Elsevier Science Publishing, New York, 1986, pp. 461-478.

[9] Ficapal A., Alonso-Urmeneta B., Velasco J., Moriyón I., Blasco J.M., Diagnosis of Brucella ovis Infection of rams with an ELISA using Protein G conjugate, Vet. Rec. 137 (1995) 145-147.

[10] Farrell I.D., The development of a new selective medium for the isolation of Brucella abortus from contaminated sources, Res. Vet. Sci. 16 (1974) 280-286.

[11] Gall D., Colling A., Marino O., Nielsen K., Perez B., Samartino L., Enzyme-immunoassays for serological diagnosis of bovine 
brucellosis: A trial in Latin America, Clin. Diagn. Lab. Immunol. 5 (1998) 654-661.

[12] Garin-Bastuji B., Blasco J.M., Grayon M., Verger J.M., Brucella melitensis infection in sheep: present and future, Vet. Res. 29 (1998) 255-274

[13] Hendry D.M.F.D., Corbel M.J., Bell R.A., Stack J.A., Brucella Antigen Production and Standardization, Central Veterinary Laboratory (CVL), New Haw, Weybridge, 1985.

[14] Jiménes De Bägues M.P., Marín C., Blasco J.M., Moriyón I., Gamazo C., An Elisa with Brucella lipopolysaccharide antigen for the diagnosis of $B$. melitensis infection in sheep and for the evaluation of serological responses following subcutaneous or conjuntival B. melitensis strain Rev 1 vaccination, Vet. Microbiol. 30 (1992) 233-241.

[15] Joint FAO/WHO Expert Commitie on Brucellosis, Sixth Report, Technical Report Series 740, WHO, Geneva, Switzerland, 1986.

[16] Kittelberg R., Bundesen P.G., Cloeckaert A., Greiser-Wilke I., Letessen J.J., Serological cross-reactivity between Brucella abortus and Yersinia enterocolytica 0:9: evaluation of the $\mathrm{M}$ and $\mathrm{C}$ epitope antibody response for the specific detection of $B$. abortus infection, Vet. Microbiol. 60 (1998) 45-57.

[17] Kittelberger R., Diack D.S., Vizcaíno N., Zigmunt M.S., Cloeckaert A., Characterization of an immuno-dominant antigen in Brucella ovis and evaluation of its use in an Enzyme-Linked Immunosorbent Assay, Vet. Microbiol. 59 (1998) 213-227.

[18] Limet J.N., Kerkhofs P., Wijffels R., Dekeyser P., Le diagnostic sérologique de la brucellose bovine par ELISA, Ann. Méd. Vét. 132 (1988) 565-575.

[19] Mahajan N.K., Kulshreshtha C.L., Comparison of serological tests for Brucella melitensis infection in sheep, Trop. Anim. Health Prod. 23 (1991) 11-16.

[20] Marín C., Alabart J.L., Blasco J.M., Effect of antibiotics contained in two brucella selective media on growth of Brucella abortus, B. melitensis and B. ovis, Vet. Rec. 34 (1996) 426-428.

[21] Marín C., Jimenéz de Bagüés M.P., Barberán M., Blasco J.M., Comparison of two selective media for the isolation of Brucella melitensis from naturally infected sheep and goats, Vet. Rec. 27 (1996) 409-411.

[22] Marin C.M., Alonso-Urmeneta B., Moriyón I., Pérez-Gómez S., Blasco J.M., Comparison of polyclonal, monoclonal and Protein $\mathrm{G}$ peroxidase conjugates in an enzyme-linked immunosorbent assay for the diagnosis of Brucella ovis in sheep, Vet. Rec. 143 (1998) 390-394

[23] Marín C., Moreno E., Moriyón I., Diáz R. Blasco J.M., Performance of competitive and indirect enzyme-linked immunosorbent assays, gel immunoprecipitation with native hapten polysaccharide and standard serological tests in diagnosis of sheep brucellosis, Clin. Diagn. Lab. Immunol. 6 (1999) 269272.

[24] Mikolon A.B., Gardner I.A., Hietala S.K., Hernandez de Anda J., Pestaña E.C., Hennager S.G., Edmondson A.J., Evaluation of north American antibody detection tests for diagnosis of brucellosis in goats, J. Clin. Microbiol. 36 (1998) 1716-1722.

[25] Moriyón I., Gamazo C., Structures and properties of the outer membrane of Brucella abortus and Brucella melitensis, Intern. Microbiol. 1 (1998) 19-26.

[26] OIE Manual of standards for diagnostic tests and vaccines OIE, Paris, France, 1996.

[27] Pilet C., Bourdon J.L., Toma B., Marchal N., Balbastre C., Person J.M., Yersinia in: Bactériologie médicale et vétérinaire - Systématique bactérienne, Doin Éditeurs, Paris, 1987, pp. 135-140.

[28] Samartino L., Gall D., Gregoret R., Nielsen K., Validation of enzyme-linked immunosorbent assays for the diagnosis of bovine brucellosis, Vet. Microbiol. 70 (1999) 193200.

[29] Sangari F.J., Aguëro J., Molecular basis of Brucella pathogenicity: an update, Microbiología SEM 12 (1996) 207-218.

[30] Vigliocco A.M., Silva Paulo P.S., Meste J., Briones G.C., Draghi G., Tossi M., Nielsen K., Development and validation of an indirect enzyme immunoassay for detection of ovine antibody to Brucella ovis, Vet. Microbiol. 54 (1997) 357-368. 